\title{
Dual reciprocity boundary element modeling of collimated light fluence distribution in normal and cancerous prostate tissue during photodynamic therapy
}

\author{
K. E. Donne, A. Marotin \& A. Al-Hussany \\ Faculty of Applied Design and Engineering, Swansea Metropolitan, \\ University of Wales Trinity Saint David, UK
}

\begin{abstract}
Modified dual reciprocity boundary element (MDBM) solution to the diffusion approximation of the radiative transport equation is employed to calculate the light distribution in normal and cancerous tissue, when irradiated with a collimated light beam via a cylindrical diffusion optical fiber. Three different active widths of the light source were investigated separately. The results of the light distribution obtained with this method were compared to results obtained using an in-house Monte-Carlo (MC) simulation program, and showed very close agreement with each other. Both methods showed that the optical penetration depth of the therapeutic light is lower in cancerous than in normal prostate tissue. The MDBM is over $90 \%$ faster than the MC technique, which could eventually be used to develop a real-time multidimensional program to (1) qualitatively estimate the therapeutic light distribution in heterogeneous tissue and (2) predict the best position of light sources in the targeted tissue to optimise photodynamic therapy treatment planning.

Keywords: photodynamic therapy, radiative transport equation, photosensitiser, dual reciprocity boundary element, geometric function, radiance, anisotropy factor, fundamental solution, phase function and fluence rate.
\end{abstract}

\section{Introduction}

Photodynamic therapy (PDT) is a relatively new emerging treatment modality for malignant (and benign) diseases that combines the administration of a certain 
chemical compound (photosensitiser) and irradiation with sufficient light dose of the proper wavelength to form, in the presence of molecular oxygen, a potentially reactive species that can cause cellular damage in a selective region [1].

Targeting the photosensitiser at the tumor effectively while minimizing the damage of the nearby healthy tissue, necessitates accurate light dosimetry planning during the PDT treatment [2]. Basically, two processes may occur when biological tissue is exposed to light (photons): scattering and absorption; the former is usually dominant in the near infrared spectral range. Scattering in biological tissue has shown to have a forward directed distribution. Malignant tumors are associated with higher blood content due to enhanced microvascularization inside or around the tumor [3], and have substantially stronger absorption coefficient in the near infrared region than all other surrounding normal tissue. In contrast, benign tumors have a normal level of blood oxygenation. This differentiation between malignant and benign tumors is the most significant property that can be assessed noninvasively. One of the key parameters to successful light therapy for solid cancer treatment (such as prostate cancer) is the accurate planning of the spatial distribution of the light dose delivered to the tumour tissue while minimising the amount of light received by the surrounding normal tissue (optimise efficacy and safety of the PDT treatment). Currently, most of the available computational models are based on diffusion theory and/or the Monte Carlo technique. In the diffusion theory, the transport of light is modelled as a gradient driven diffusion of energy [4], while the Monte Carlo technique models the light as discrete photon packets bouncing around and being absorbed throughout the scattering medium [5]. In this paper, the fluence rate of the light distribution delivered to the normal and cancerous prostate is estimated numerically using a modified Dual Reciprocity Boundary Element Method and the results are compared with that obtained using an in house Monte Carlo simulation code. The method is based on a modified 3D BEM code developed by Donne et al. [6]. The present study is the framework for a long term project aiming to develop an accurate real-time multidimensional numerical model to qualitatively estimate the light dosimetry in heterogeneous tissue and optimise the PDT treatment planning for prostate cancer.

\section{Modeling of light transport in biological tissue}

Light propagation in biological tissue is often modelled based on the diffusion approximation to the Radiative Transport Equation (RTE) [7], and is given by

$$
\frac{1}{c} \frac{\partial \emptyset_{d}(r, t)}{\partial t}+D \Delta \emptyset_{d}(r, t)-\mu_{a} \phi_{d}(r, t)=-Q_{0}(r, t)
$$

where $\emptyset_{d}$ is the radiant energy fluence rate $\left(\mathrm{W} \cdot \mathrm{m}^{-2}\right), c$ is the speed of the light in the medium, $Q_{0}$ represents an isotropic source and $D$ is the diffusion coefficient [8]. 
In the case of PDT treatment of prostate cancer where a variable width of collimated light beam is used, the diffusion equation cannot provide accurate and reliable results. This problem was investigated by Donne et al. [6] who used a BEM to model the distribution of a collimated beam of light in biological tissue. Donne et al. introduced a new geometric function (based on the normalised Henyey-Greenstein phase function), which describes the relationship between an incident collimated beam of light on the boundary surface and the angular probability of the scattered beam distribution propagating inside the medium. The geometric function is of the form

$$
\chi(\eta)=\frac{\beta^{3}}{\left(\beta^{2}-2(\beta-1)(1-\eta)\right)^{\frac{3}{2}}}
$$

Such that $\int_{0}^{4 \pi} \chi(\eta) d \eta=0$, where $\eta=\cos (\theta)$, with $\theta$ being the angle between the centre of the domain element and an irradiated boundary node. $\beta$ is a parameter which describes the degree of isotropic scattering and its value varies between 0 and 1. A reasonable approximation of tissue scattering can be obtained by choosing a suitable value $\beta$ in the function $X(\eta)$ without altering the boundary conditions or requiring any buried source terms.

In this paper we explore the modification developed by Donne et al. [6] and the Dual Reciprocity Boundary Element Method (DBM) $[9,10]$ to compute the fluence rate distribution of light in normal and cancerous prostate tissue.

\section{Numerical solution of the diffusion equation}

Considering no sources, eqn. (1) can be written as

$$
D \nabla^{2} \emptyset_{d}(r, t)=b\left(\phi_{d}(r, t), \frac{\partial \emptyset_{d}(r, t)}{\partial t}\right)
$$

The equivalent dual reciprocity boundary integral equation to the differential eqn. (3) can be expressed as the sum of the solution of the Laplace's equation (homogeneous) and a series of particular solutions $\widehat{\emptyset}_{j}$ instead of a single particular solution $\widehat{\emptyset}$ for the non-homogeneous part of eqn. (3) [10]

$$
\nabla^{2} \widehat{\varnothing}=b\left(\phi_{d}, \dot{\emptyset}_{d}\right)
$$

The non-linear term in eqn. (3) can be expressed as a combination of two parts,

$$
b\left(\emptyset_{d}\right)=\sum_{j=1}^{N+L} \alpha_{j} f_{j}(x, y) \text { and } b\left(\frac{\partial \emptyset_{d}}{\partial t}\right)=\sum_{j=1}^{N+L} \beta_{j}(t) f_{j}(x, y)
$$

where the expansion function $f_{j}$ is geometry dependent and related to the particular solution $\widehat{\emptyset}_{j}$ through the relation $f_{j}=\nabla^{2} \widehat{\emptyset}_{j} . N$ and $L$ are the number of boundary and internal nodes respectively, $\alpha_{j}$ are unknown coefficients and $\beta_{j}(t)$ are time dependent unknown coefficients. Eqn. (3) can be written as

$$
\frac{1}{c} \sum_{j=1}^{N+L} \beta_{j}(t) \nabla^{2} \widehat{\emptyset}_{j}+\mathrm{D} \nabla^{2} \emptyset_{d}(r, t)=\mu_{a} \sum_{j=1}^{N+L} \alpha_{j} \nabla^{2} \widehat{\emptyset}_{j}
$$


Multiplying eqn. (5) by the fundamental solution of the Laplace equation [11], integrating over the domain then applying the green's theorem, we get the following integral equation for each node $i$

$$
\begin{gathered}
\frac{1}{c} \sum_{j=1}^{N+L} \beta_{j}(t)\left\{C(i) \widehat{\emptyset}(i)+\int_{\Gamma} \widehat{\emptyset}_{j} q^{*} d \Gamma-\int_{\Gamma} \emptyset_{d}^{*} \hat{q}_{j} d \Gamma\right\}+C(i) \emptyset_{d}(i)+\int_{\Gamma} \emptyset_{d} q^{*} d \Gamma \\
-\int_{\Gamma} \emptyset_{d}^{*} q d \Gamma=\mu_{a} \sum_{j=1}^{N+L} \alpha_{j}\left\{C(i) \widehat{\emptyset}(i)+\int_{\Gamma} \widehat{\emptyset}_{j} q^{*} d \Gamma-\int_{\Gamma} \emptyset_{d}^{*} \hat{q}_{j} d \Gamma\right\}
\end{gathered}
$$

The free term $C(i)$ is typically 0.5 at a smooth boundary point, $q^{*}$ is the normal derivative of the fundamental solution [11]

$$
\phi_{d}^{*}(i, j)=\frac{1}{4 \pi D r_{i j}} e^{-\mu_{e f f} r_{i j}}
$$

where $r_{i j}$ is the distance between the source point $i$ and the field point $j$, and $\mu_{e f f}=\sqrt{\frac{\mu_{e f f}}{D}}$ is the effective attenuation coefficient. In terms of nodal value, after discretisation of the surface $\Gamma$ into $N$ boundary elements [12], eqn. (6) can be written

$$
\begin{gathered}
\frac{1}{c}\left(\sum_{j=1}^{N+L} \beta_{j}(t)\left\{c_{i j} \widehat{\emptyset}_{i j}+\sum_{k=1}^{N} \widehat{H}_{i k} \widehat{\emptyset}_{k j}-\sum_{k=1}^{N} G_{i k} \widehat{q}_{k j}\right\}\right)+D\left(c_{i} \emptyset_{i}+\sum_{k=1}^{N} \widehat{H}_{i k} \emptyset_{k}\right. \\
\left.-\sum_{k=1}^{N} G_{i k} q_{k}\right)=\mu_{a}\left(\sum_{j=1}^{N+L} \alpha_{j}\left\{c_{i j} \widehat{\emptyset}_{i j}+\sum_{k=1}^{N} \widehat{H}_{i k} \widehat{\emptyset}_{k j}-\sum_{k=1}^{N} G_{i k} \widehat{q}_{k j}\right\}\right)
\end{gathered}
$$

where $\widehat{H}_{i k}$ and $G_{i k}$ are called the influence coefficients such as,

$$
\widehat{H}_{i k}=\int_{\Gamma_{j}} q^{*}(i, k) d \Gamma \quad \text { and } \quad G_{i k}=\int_{\Gamma_{j}} \phi_{d}^{*}(i, k) d \Gamma
$$

The term $\widehat{\emptyset}_{j}$ can be obtained by integration $f_{j}=\nabla^{2} \widehat{\emptyset}_{j} \quad$ [10]. After application to all boundary nodes and using a collection technique, eqn. (8) can be expressed in the following matrix form

$$
(\mathrm{H} \widehat{U}-\mathrm{G} \widehat{\mathrm{Q}}) \beta(t)=D c\left(\mathrm{H} \emptyset_{d}-\mathrm{Gq}\right)-c \mu_{a}(\mathrm{H} \widehat{\mathrm{U}}-\mathrm{G} \widehat{\mathrm{Q}}) \alpha
$$

where $\mathrm{H}$ and $\mathrm{G}$ are two $N \times N$ square matrices, $\emptyset_{d}$ and $q$ are vectors of length $N$. The unknown coefficients $\alpha_{j}$ and $\beta_{j}$ can be written in matrix form as $\alpha=$ $F^{-1} \emptyset_{d}$ and $\beta=F^{-1} \frac{\partial \emptyset_{d}}{\partial t}$. The matrix $F$ of length $(N+L) \times(N+L)$ depends only on geometric data. Eqn. (9) becomes

$$
(\mathrm{H} \widehat{\mathrm{U}}-\mathrm{G} \widehat{\mathrm{Q}}) \mathrm{F}^{-1} \frac{\partial \emptyset_{d}}{\partial t}=D c\left(\mathrm{H} \emptyset_{d}-\mathrm{Gq}\right)-c \mu_{a}(\mathrm{H} \widehat{\mathrm{U}}-\mathrm{G} \widehat{\mathrm{Q}}) \mathrm{F}^{-1} \emptyset_{d}
$$

Defining a new matrix, $S=(H \widehat{U}-G \widehat{Q}) F^{-1}$, eqn. (10) after rearranging can be written as

$$
\frac{\partial \emptyset_{d}}{\partial t}=\left(D c \mathrm{H}-c \mu_{a} \mathrm{~S}\right) \mathrm{S}^{-1} \emptyset_{d}-D c \mathrm{GqS} \mathrm{S}^{-1}
$$

Robin Boundary Condition (RBC) defines a more realistic approach to solve eqn. (3), as it represents a non-scattering-back medium exterior to the boundary [13]. 
The RBC can be modified to incorporate a mismatch between the refractive indices $n_{\Omega}$ within $\Omega$ and $n^{\prime}$ in the surrounding medium. Incorporating the diffuse source as a photon current directly through the boundary $\Gamma_{2}$ as shown in Figure 1, the RBC boundary condition can be described as $\frac{\partial \phi(\xi)}{\partial n}=\psi(\xi) \phi(\xi)+$ $g(\xi)$ with

$$
\left.\left.\begin{array}{c}
\psi(\xi)=\frac{-1}{2 D A} \\
g(\xi)=0
\end{array}\right\} \forall \xi \in \Gamma_{1} \quad \text { and } \quad \begin{array}{c}
\psi(\xi)=\frac{-1}{2 D A} \\
g(\xi)=\frac{-2 F_{d s}}{D A}
\end{array}\right\} \forall \xi \in \Gamma_{2}
$$

where $F_{d s}$ is the strength of the source current, and $A=\frac{\frac{2}{1-R_{0}}-1+\left|\cos \theta_{c}\right|^{3}}{1-\left|\cos \theta_{c}\right|^{2}}$. Eqn. (11) represents a steady state of the radiative transport equation when $\frac{\partial \emptyset_{d}}{\partial t}=0$

$$
\left(D H-\mu_{a} S\right) \emptyset_{d}=D G q
$$

Applying the boundary conditions described by eqn. (12), and using the geometrical function given by eqn. (2), we obtained the following system of equations

$$
\left.\begin{array}{lr}
\left(D H-\mu_{a} S\right) \emptyset_{d}=0 & l \& j \in \Omega \\
\left(D H-\mu_{a} S-D \psi G\right) \emptyset_{d}=0 & l \in \Omega \& j \in \Gamma_{1} \\
\left(D H-\mu_{a} S-D \psi G\right) \emptyset_{d}=G\left(\frac{-2 F_{d s}}{D A}\right) & l \in \Gamma_{1} \& j \in \Gamma_{1} \\
\left(D H-\mu_{a} S-D \psi G\right) \chi \emptyset_{d}=\chi G\left(\frac{-2 F_{d S}}{D A}\right) & l \in \Omega \& j \in \Gamma_{1}
\end{array}\right\}
$$

The unknown $N$ boundary and $L$ internal values of $\phi_{d}$ are coupled and obtained simultaneously when solving eqn. (14) as $\boldsymbol{A} \boldsymbol{X}=\boldsymbol{B}$, where the vector $\boldsymbol{X}$ contains $N+L$ unknown values of $\phi_{d}$. In this paper, the internal points were chosen to cover the whole domain; hence, there is no need for the internal solutions.

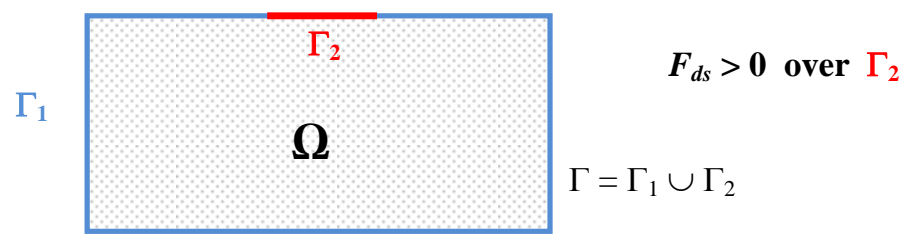

Figure 1: Collimated light source on the boundary.

\section{Modelling of collimated light fluence distribution in normal and cancerous tissue}

In order to reduce unnecessary computations during simulation, only 20-mm (axial) by $20-\mathrm{mm}$ (lateral) regions of a tissue sample representing a human prostate (normal or cancerous prostate) were visualised in all computational 
experiments. The sample was assumed to be irradiated with light beams of wavelength $625 \mathrm{~nm}$ and active widths of 20, 15 and $7.5 \mathrm{~mm}$ respectively. The distribution of the absorbed energy from the beam within the sample were estimated numerically using the following methods; a) conventional Dual boundary element method (CDBM), where $X(\eta)=1$ in eqn. (14), b) modified Dual boundary element method (MDBM), and c) Monte Carlo simulation technique (MC). The optical transport properties $\left(\mu_{s}, \mu_{a}, \mathrm{n}\right.$, and $\left.\mathrm{g}\right)$ of the normal prostate tissue were chosen to be $16 \mathrm{~mm}^{-1}, 0.07 \mathrm{~mm}^{-1}, 1.4$ and 0.9 respectively [13]. The differentiation between malignant and normal prostate tissue was based on the absorption coefficient where the concentration of strongly absorbing molecules such as haemoglobin and other porphyrins are increased many fold in the malignant tissues. Therefore, the optical transport properties of cancerous prostate were chosen to be $16 \mathrm{~mm}^{-1}, 0.2 \mathrm{~mm}^{-1}, 1.4$ and 0.9 for $\mu_{s}, \mu_{a}$, n, and $\mathrm{g}$ respectively. The energy of the light beam was fixed to $50 \mathrm{~J} / \mathrm{cm}^{2}$, and the light was uniformly distributed over the irradiated boundary area. The domain boundary was discretised with 160 linear elements of constant width and the 2D domain was discretised with 3586 triangular elements.

Figures 2-4 show the profiles of the absorbed energy distribution (fluence) in the normal and cancerous tissue (the color scale is: red $\left(50 \mathrm{~J} / \mathrm{cm}^{2}\right)$, gold-orange $\left(25 \mathrm{~J} / \mathrm{cm}^{2}\right)$, yellow $\left(5 \mathrm{~J} / \mathrm{cm}^{2}\right)$ and green $\left(1 \mathrm{~J} / \mathrm{cm}^{2}\right)$ ) as calculated with MC, MBEM and CBEM respectively. Comparison between the MC and the MDBM results shows that both methods provide comparable results with respect to the distribution of the absorbed energy in the biological tissue where the propagated light spread and reach deeply into the tissue. However, the computation time of the MBEM approach was approximately 1\% that of the MC approach for $10^{7}$ photons. The pattern is different with the CDBM approach, where the energy

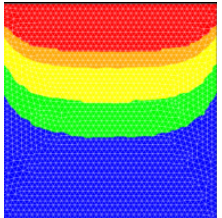

(a)

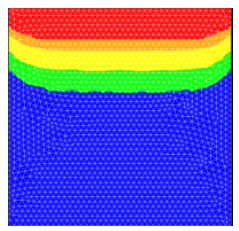

(d)

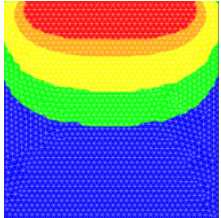

(b)

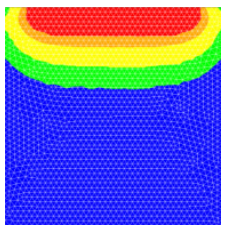

(e)

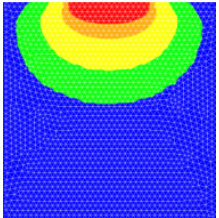

(c)

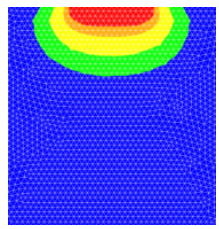

(f)

Figure 2: $\quad \mathrm{CM}$ simulation of the internal photon density distribution within normal tissue (a)-(c) and tumor tissue (d)-(f) for illuminated segment $\Gamma_{2}=20 \mathrm{~mm}$ (a) and (d), $\Gamma_{2}=15 \mathrm{~mm}$ (b) and (e), and $\Gamma_{2}=7.5 \mathrm{~mm}(\mathrm{c})$ and (f). 


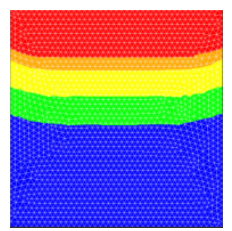

(a)

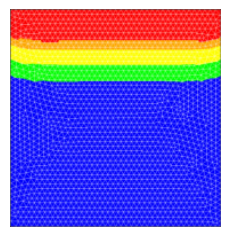

(d)

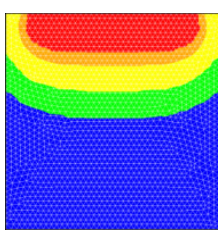

(b)

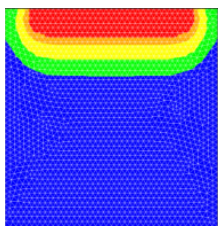

(e)

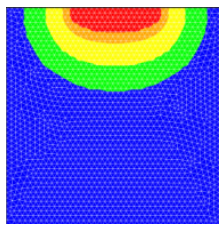

(c)

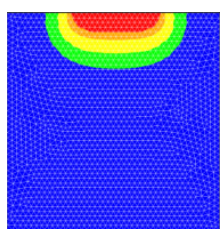

(f)

Figure 3: MDBM calculation of the internal photon density distribution within normal tissue (a)-(c) and tumor tissue (d)-(f) for illuminated segment $\Gamma_{2}=20 \mathrm{~mm}$ (a) and (d), $\Gamma_{2}=15 \mathrm{~mm}$ (b) and (e), and $\Gamma_{2}=7.5 \mathrm{~mm}$ (c) and (f).

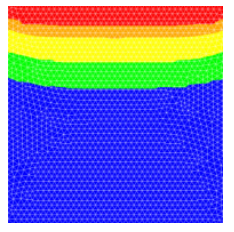

(a)

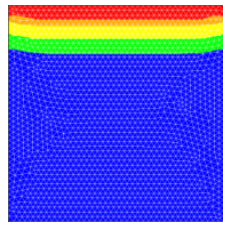

(d)

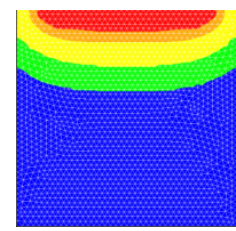

(b)

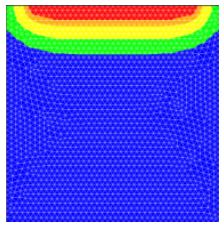

(e)

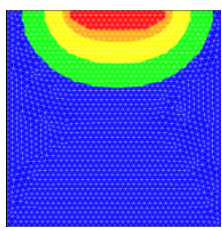

(c)

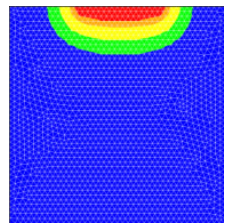

(f)

Figure 4: CDBM calculation of the internal photon density distribution within normal tissue (a)-(c) and tumor tissue (d)-(f) for illuminated segment $\Gamma_{2}=20 \mathrm{~mm}$ (a) and (d), $\Gamma_{2}=15 \mathrm{~mm}$ (b) and (e), and $\Gamma_{2}=7.5 \mathrm{~mm}$ (c) and (f).

distribution inside the prostate is concentrated near the surface and is quickly dissipated away as it goes deeper into the sample. Consequently, this approach cannot provide accurate and reliable information regarding the distribution of therapeutic light used in PDT.

Figure 5 shows the results of the normalised energy deposited along the direction of the incident beam within the domain (vertical axis of symmetry) as 
calculated by the three numerical approaches for normal and cancerous tissue and for $20 \mathrm{~mm}$ and $15 \mathrm{~mm}$ light beam widths respectively. It is clear that the boundary element method as adopted and modified by Donne et al. [6] provides comparable results to the reliable and computationally expensive MC method.

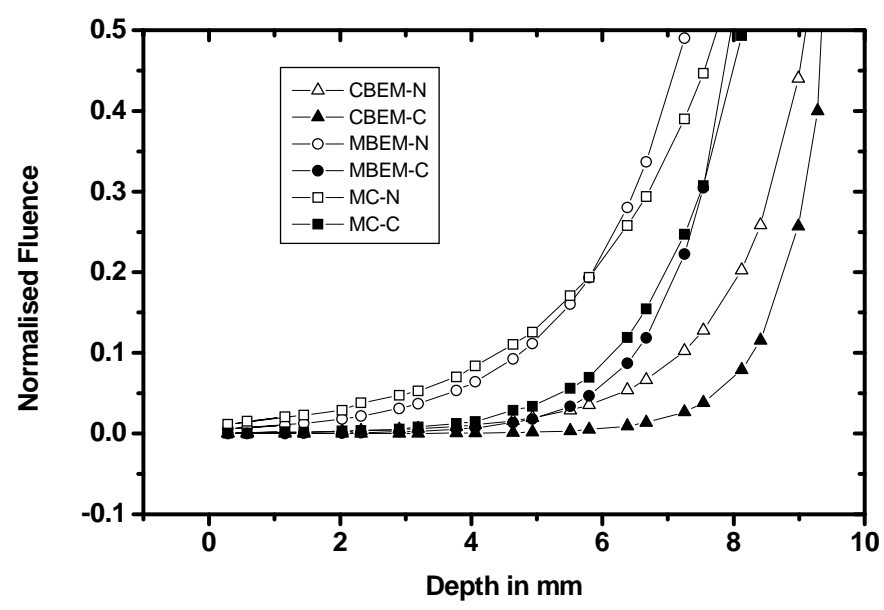

(a)

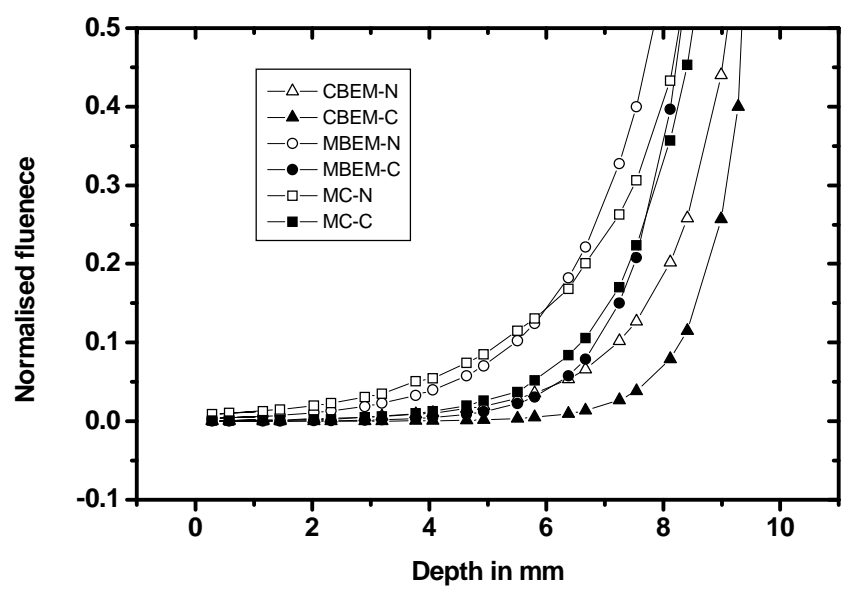

(b)

Figure 5: Energy deposition along the central propagation path of a collimated beam of light of active length $20 \mathrm{~mm}$ (a) and $15 \mathrm{~mm}$ (b) illuminating the surface of normal $(\mathrm{N})$ and cancerous tissue $(\mathrm{C})$ obtained by MC, MDBM and CDBM. The surface illuminated is located at depth $\mathrm{y}=10 \mathrm{~mm}$. 
Table 1 summarises the position $(\mathrm{mm})$ of the isofluence lines or lines of equal fluence of 50, 25, 5 and $1 \mathrm{~J} / \mathrm{cm}^{2}$ along the central propagation path in normal and cancerous prostate as calculated by MC simulation, MDBM, and CDBM. The differences between the position of the isofluence lines predicted by MC and modified BEM are always less than 9\%. However, this is not the case for lower isofluence lines of 5 and $1 \mathrm{~J} / \mathrm{cm}^{2}$ where the differences go up to nearly $14 \%$. The differences between the isofluence lines position predicted by MDBM and CDBM of 50 and $25 \mathrm{~J} / \mathrm{cm}^{2}$ go up to $57 \%$, which indicate that the CDBM underestimates the optical penetration depth inside the biological tissue. This could have potential effects on the therapeutic treatment plan.

Table 1: Position $(\mathrm{mm})$ of the isofluence lines $\left(\mathrm{J} / \mathrm{cm}^{2}\right)$ along the central propagation path for light beams of different active lengths: (a) $20 \mathrm{~mm}$ (b) $15 \mathrm{~mm}$ and (c) $7.5 \mathrm{~mm}$. Normal tissue (NT) and cancerous tissue (CT).

\begin{tabular}{|c|c|c|c|c|c|c|c|c|}
\cline { 2 - 9 } \multicolumn{1}{c|}{} & \multicolumn{2}{c|}{$50 \mathrm{~J} / \mathrm{cm}^{2}$} & \multicolumn{2}{c|}{$25 \mathrm{~J} / \mathrm{cm}^{2}$} & \multicolumn{2}{c|}{$5 \mathrm{~J} / \mathrm{cm}^{2}$} & \multicolumn{2}{c|}{$1 \mathrm{~J} / \mathrm{cm}^{2}$} \\
\cline { 2 - 9 } \multicolumn{1}{c|}{} & NT & CT & NT & CT & NT & CT & NT & CT \\
\hline MC & 2.57 & 1.77 & 3.37 & 2.32 & 5.12 & 3.10 & 6.85 & 4.15 \\
\hline MBEM & 2.55 & 1.75 & 3.25 & 2.25 & 4.62 & 3.15 & 6.15 & 3.9 \\
\hline CBEM & 1.15 & 0.75 & 1.75 & 1.15 & 3.15 & 1.8 & 4.5 & 2.4 \\
\hline
\end{tabular}

(a)

\begin{tabular}{|c|c|c|c|c|c|c|c|c|}
\cline { 2 - 9 } \multicolumn{1}{c|}{} & \multicolumn{2}{c|}{$50 \mathrm{~J} / \mathrm{cm}^{2}$} & \multicolumn{2}{c|}{$25 \mathrm{~J} / \mathrm{cm}^{2}$} & \multicolumn{2}{c|}{$5 \mathrm{~J} / \mathrm{cm}^{2}$} & \multicolumn{2}{c|}{$1 \mathrm{~J} / \mathrm{cm}^{2}$} \\
\cline { 2 - 9 } \multicolumn{1}{c|}{} & N.T & C.T & N.T & C.T & N.T & C.T & N.T & C.T \\
\hline MC & 2.08 & 1.66 & 3.12 & 2.17 & 4.73 & 3.15 & 5.81 & 4.05 \\
\hline MBEM & 2.10 & 1.62 & 2.85 & 2.13 & 4.30 & 3.05 & 5.25 & 3.83 \\
\hline CBEM & 1.12 & 0.75 & 1.72 & 1.10 & 3.07 & 1.72 & 4.41 & 2.35 \\
\hline
\end{tabular}

(b)

\begin{tabular}{|c|c|c|c|c|c|c|c|c|}
\cline { 2 - 9 } \multicolumn{1}{c|}{} & \multicolumn{2}{c|}{$50 \mathrm{~J} / \mathrm{cm}^{2}$} & \multicolumn{2}{c|}{$25 \mathrm{~J} / \mathrm{cm}^{2}$} & \multicolumn{2}{c|}{$5 \mathrm{~J} / \mathrm{cm}^{2}$} & \multicolumn{2}{c|}{$1 \mathrm{~J} / \mathrm{cm}^{2}$} \\
\cline { 2 - 9 } \multicolumn{1}{c|}{} & N.T & C.T & N.T & C.T & N.T & C.T & N.T & C.T \\
\hline MC & 1.25 & 1.05 & 1.92 & 1.60 & 3.72 & 2.52 & 5.35 & 3.40 \\
\hline MBEM & 1.30 & 1.10 & 1.85 & 1.52 & 3.35 & 2.30 & 4.65 & 3.18 \\
\hline CBEM & 1.08 & 0.72 & 1.68 & 1.08 & 2.90 & 1.75 & 4.38 & 2.40 \\
\hline
\end{tabular}

(c) 


\section{Discussion}

The tumor microenvironment is highly heterogeneous and hypoxic. During PDT treatment, the heterogeneity of the hypoxia distribution is exacerbated. Lowering the PDT radiant fluence rate reduces the rate of oxygen consumption, the heterogeneity created by hypoxia, and also the heterogeneity in cytotoxic response. In contrast, high fluence rates can lead to significant and direct tumor cell destruction. The treatment must be carried under oxygen conserving conditions, otherwise, high fluence rates will induce acute hypoxia and limiting treatment effectiveness. Therefore, the core of any PDT planning treatment is modeling, optimizing and calculation of the light distribution in tissue generated by the therapeutic light beam of variable active length.

The computational results carried out in this work show that the higher absorption coefficient of the tumor leads to lower penetration depths and higher energy densities in the tumor. In contrast, the optical penetration depth is higher and the energy density is lower in healthy tissue (as shown in figures 2-4). This indicates that the levels of light energy that can reach and activate the photosensitiser located inside the tumor to create the desired photodynamic effect are limited to smaller areas of the tumor directly surrounding the light beam. To overcome the low penetration depth in prostate tumors, it is necessary to either increase the dose of the therapeutic light or apply it for longer times (which in this case has a profound effect on healthy tissue surrounding the tumor), or repeat the treatment application to effectively treat the entire tumor.

When illuminating the tissue with a collimated beam of light, the diffusion equation is not a good approximation of the transport theory compared to the MC technique as illustrated in figures 2 and 4, and table 1. This deficiency was rectified by Donne et al. [6] by introducing a geometric function describing the relationship between an incident collimated beam of light on the boundary surface and the angular probability of the scattered beam distribution propagating inside the medium. In this framework, the light distribution in tumor and normal prostate tissue were qualitatively predicted numerically using the MDBM developed by the same authors and are illustrated in figures 3 and 5, and table 1 .

The MDBM appears to be the most appropriate method for evaluating the light distribution through biological tissues with different absorption coefficients. It provides comparable results to the MC technique with computation time savings of up to $90 \%$.

\section{Conclusion}

For a given mesh, the therapeutic light distribution predicted by the MDBM gives comparable results to the Monte-Carlo method which is renowned for its accuracy and flexibility but requires long computational times. The MDBM takes into consideration the deficiencies of the most conventional numerical solutions of the diffusion equation such as the finite difference, the finite element and the boundary element techniques. The MDBM is more appropriate and 
realistic than the current approximation where fictitious buried isotropic sources are introduced in the tissue to represent a collimated beam. The results of this study motivate us to propose a multidimensional program to qualitatively estimate the therapeutic light distribution in heterogeneous tissue.

\section{References}

[1] Moore C. M., Nathan T. R., Lees W. R., Mosse C. A., Freeman A., Emberton M. and Bown S. G., "Photodynamic therapy using meso tetra hydroxyl phenyl chlorine (mTHPC) in early prostate cancer”, Lasers Surg. Med., 38, 356-63, 2006.

[2] Henderson B. and Dougherty T., "How does photodynamic therapy work?”, Photo chemistry and Photobiology, 55(1), pp. 145-157, 1992.

[3] Mukherjee P., Sotnikov A.V., Mangian H. J., Zhou J. R., Visek W. J., Clinton S. K., "Energy intake and prostate tumor growth, angiogenesis, and vascular endothelial growth factor expression,” J. NCI , 91(6): 512-523, 1999.

[4] Jacques S. and Pogue B., "Tutorial on diffuse light transport”, Journal of Biomedical Optics, 13(4), (041302), 2008.

[5] Welch A. J. and Van Gemert M. J. C., Optical-thermal response of laserirradiated tissue, New York: Plenum Press, 1995.

[6] Donne K. E., Marotin A., Al-Hussany A. and Daniel G. M., "Modified Boundary Element to Model Radiative Transport in Biological Turbid Medium”, International Journal of Engineering simulation, IJES 10th Anniversary Issue, 2011.

[7] Van Gemert M. J. C., Jacques S. L., Sterenborg H. J. C. M. and Star W. M., “Skin optics”, IEEE Trans. Biomed. Eng., 36(12), pp. 1146-1154, 1989.

[8] Wang L. V. and Jacques S. L., "Source of error in calculation of optical diffuse reflectance from turbid media using diffusion theory”, Comput. Meth. Prog. Biomed., 61,pp. 163-170, 2000.

[9] Trevelyan J., Boundary Element for Engineers - Theory and Applications, Southampton: Computational Mechanics Publications, 1994.

[10] Partridge P.W., Brebbia C. A. and Wrobel L.C., The Dual Reciprocity Boundary Element Method, Computational Mechanics Publications, Southampton, London, 1992.

[11] Hielscher A. H., Alcouffe R. E. and Barbour R. L., "Comparison of finitedifference transport and diffusion calculations for photon migration in homogeneous and heterogeneous tissues”, Phys. Med. Biol., 43(5), pp. 1285-1302, 1998.

[12] Wrobel L. C., The Boundary Element Method - Volume 1 - Applications in Thermo-Fluids and Acoustics, Chichester: John Wiley \& Sons, 2002.

[13] Marotin A., An Investigation into the Boundary Element Method for MultiDimensional Modelling of Light-Tissue Interaction., PhD Thesis, Swansea Metropolitan University, 2007. 